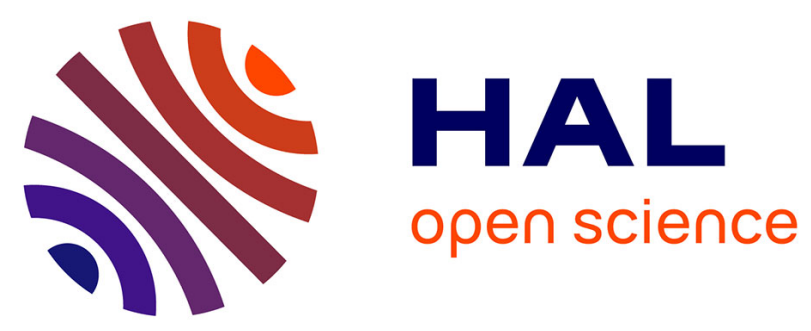

\title{
RECOVERING NAVIER-STOKES EQUATIONS FROM ASYMPTOTIC LIMITS OF THE BOLTZMANN GAS MIXTURE EQUATION
}

Carlo Bianca, Christian Dogbe

\section{- To cite this version:}

Carlo Bianca, Christian Dogbe. RECOVERING NAVIER-STOKES EQUATIONS FROM ASYMPTOTIC LIMITS OF THE BOLTZMANN GAS MIXTURE EQUATION. Communications in Theoretical Physics, 2016. hal-02151781

\section{HAL Id: hal-02151781 \\ https://hal.science/hal-02151781}

Submitted on 10 Jun 2019

HAL is a multi-disciplinary open access archive for the deposit and dissemination of scientific research documents, whether they are published or not. The documents may come from teaching and research institutions in France or abroad, or from public or private research centers.
L'archive ouverte pluridisciplinaire HAL, est destinée au dépôt et à la diffusion de documents scientifiques de niveau recherche, publiés ou non, émanant des établissements d'enseignement et de recherche français ou étrangers, des laboratoires publics ou privés. 


\title{
RECOVERING NAVIER-STOKES EQUATIONS FROM ASYMPTOTIC LIMITS OF THE BOLTZMANN GAS MIXTURE EQUATION
}

\author{
CARLO BIANCA AND CHRISTIAN DOGBE
}

\begin{abstract}
This paper is devoted to the derivation of macroscopic fluid dynamics from the Boltzmann mesoscopic dynamics of a binary mixture of hard-sphere gas particles. Specifically the hydrodynamics limit is performed by employing different time and space scalings. The paper shows that, depending on the magnitude of the parameters which define the scaling, the macroscopic quantities (number density, mean velocity and local temperature) are solution of the acoustic equation, the linear incompressible Euler equation and the incompressible Navier-Stokes equation. The derivation is formally tackled by the recent moment method proposed by Bardos et al. [3] and the results generalize the analysis performed in [4].
\end{abstract}

Keywords: Gas mixtures; Hydrodynamic limit, Asymptotic limit, Fluid equation, Conservation law.

AMS Subject Classification (2010): 82C40, 76P05, 35Q35

\section{INTRODUCTION}

The mathematical derivation of the fluid mechanics equations from the dynamics described at the mesoscopic scale is a hard challenge considering that the hydrodynamic limit techniques require assumptions and tools of nonlinear analysis. The most famous method for deriving the fluid dynamic description from kinetic equation is based on the definition of two different scalings (parabolic/diffusive and hyperbolic) and subsequently on the so-called small Knudsen number $\varepsilon$ (proportional to the mean free path) limit, which corresponds to enhance the kinetic collision operator, responsible for the trend to the thermodynamical equilibrium, at a factor $1 / \varepsilon$. Specifically, in the diffusive scaling, a time scaling of the same order than the scaling of the collision operator is considered; the asymptotic analysis leads to parabolic equations (see, among others, papers [11], [21] and the references cited therein) and specifically to the incompressible Navier-Stokes equations. The hyperbolic scaling leads to conservation laws (or balance laws if source terms appear) [8]. The derivation of the macroscopic equations is based on the moment method which, differently from the the Hilbert and Chapman-Enskog expansion, consists in passing to the limit when the Knudsen number vanishes in the local conservation laws of mass, momentum and energy. It is worth stressing that, to the best of our knowledge, the moment method dates back to Bardos et al. in [3].

In this context, it has been observed (see [22]) that if the Knudsen number Kn and the Mach number go to zero with the same speed in $\epsilon$, the Reynolds number Re (which is the ratio between the transport and viscosity effects) remains finite. Accordingly, the following interpretation of the scaling can be considered: by scaling space and time as $\epsilon^{-1}$ and $\epsilon^{-2}$ and the velocity field as $\epsilon$, the incompressible Navier-Stokes equations are unchanged. In particular in [3], under suitable technical assumptions, it has been proved that the incompressible Navier-Stokes equations are asymptotic limit of the Boltzmann equation, see also [19]. 
This paper is concerned with the derivation of macroscopic fluid dynamics from the mesoscopic dynamics of a gas-particle system composed of two subsystems L and $\mathrm{H}$ of particles that collide elastically (binary gaseous mixture) and having masses $m^{\mathrm{L}}$ and $m^{\mathrm{H}}$. The related distribution functions $F^{\mathrm{L}}=F^{\mathrm{L}}(t, x, v)$ and $F^{\mathrm{H}}=F^{\mathrm{H}}(t, x, v)$, where $t \geqslant 0$ is the time variable, $x \in \mathbb{R}^{3}$ is the space variable, and $v \in \mathbb{R}^{3}$ the velocity variable, are thus solutions of the following Boltzmann equations system:

$$
\begin{aligned}
& \partial_{t} F^{\mathrm{L}}+v \cdot \nabla_{x} F^{\mathrm{L}}=Q\left(F^{\mathrm{L}}, F^{\mathrm{L}}\right)+Q\left(F^{\mathrm{H}}, F^{\mathrm{L}}\right) \\
& \partial_{t} F^{\mathrm{H}}+v \cdot \nabla_{x} F^{\mathrm{H}}=Q\left(F^{\mathrm{L}}, F^{\mathrm{H}}\right)+Q\left(F^{\mathrm{H}}, F^{\mathrm{H}}\right)
\end{aligned}
$$

where $Q\left(F^{\alpha}, F^{\alpha}\right)$ and $Q\left(F^{\ell}, F^{\ell}\right)$, for $\{\alpha, \ell\} \in\{L, H\}$, are the collisional integral operators which model the collisions among particles of the same subsystem (self-collisions), while $Q\left(F^{\alpha}, F^{\ell}\right)$ is the collisional term which model the collisions among the particles of the two subsystems $\alpha$ and $\ell$ (cross-collisions) and it reads:

$$
Q\left(F^{\alpha}, F^{\ell}\right)(v)=\iint_{\mathbb{R}^{3}} \int_{\mathbb{S}^{2}}\left[F^{\alpha}\left(v_{*}^{\prime}\right) F^{\ell}\left(v^{\prime}\right)-F^{\alpha}\left(v_{*}\right) F^{\ell}(v)\right] B^{\ell \alpha}(\omega \cdot \mathbf{v},|\mathbf{v}|) d v_{*} d \omega,
$$

$F\left(v_{*}^{\prime}\right)=F\left(t, x, v_{*}^{\prime}\right), \quad F\left(v^{\prime}\right)=F\left(t, x, v^{\prime}\right), \quad F\left(v_{*}\right)=F\left(t, x, v_{*}\right), \quad F(v)=F(t, x, v)$,

where $v, v_{*}$ are the pre-collisional velocity, $v^{\prime}, v_{*}^{\prime}$ are the post-collisional velocity, where $\mathbb{S}^{2}$ is the $2 d$ sphere in $\mathbb{R}^{3}, \omega$ is an arbitrary unit vector, $d \omega$ is the surface measure on it, $\mathbf{v}:=v_{*}-v$ is the relative velocity, $d \omega$ is the solid angle element in the direction of $\omega$, $B^{\ell \alpha}$ is the nonnegative differential-scattering cross section [9]. In particular in the present paper, following Chapman and Cowling [10], we consider as $B^{\ell \alpha}$ the collision kernel that corresponds to the hard-sphere gas case (cross-section):

$$
B^{\ell \alpha}=\frac{1}{2 \sqrt{2 \pi}}\left(\frac{d^{\alpha}+d^{\ell}}{2}\right)^{2}\left|\left(v_{*}-v\right) \cdot \omega\right| \quad \omega \in \mathbb{S}^{2},
$$

where $d^{\alpha}$ and $d^{\ell}$ are the atomic diameters of the particles of the two subsystems $\alpha$ and $\ell$. The momentum and energy conservation equations

$$
m^{\alpha} v^{\prime}+m^{\ell} v_{*}^{\prime}=m^{\alpha} v+m^{\ell} v_{*}, \quad m^{\alpha}\left|v^{\prime}\right|^{2}+m^{\ell}\left|v_{*}^{\prime}\right|^{2}=m^{\alpha}|v|^{2}+m^{\ell}\left|v_{*}\right|^{2},
$$

allow to derive the post-collisional velocities $v^{\prime}$ and $v_{*}^{\prime}$ :

$$
v^{\prime}=v+\frac{2 m^{\ell}}{m^{\alpha}+m^{\ell}}(\omega \cdot \mathbf{v}) \omega, \quad v_{*}^{\prime}=v_{*}-\frac{2 m^{\alpha}}{m^{\alpha}+m^{\ell}}(\omega \cdot \mathbf{v}) \omega .
$$

The interested reader in the formal derivation of the above system is referred to Kogan [18], Chapman and Cowling [10], Ferziger and Kaper [13], Hirschfelder et al. [17].

The outline of the present paper is as follows. After this introduction, Section 2 provides the necessary background on the Boltzmann equations system for the binary mixture of hard-sphere gas, including the main properties of the collisional operator and the linearized Boltzmann operator. The hydrodynamic equations are derived in Section 3 which also contains our main result. Section 4 is concerned with the proof of the main result. Finally Section 5 deals with a critical analysis and future research perspectives including the possible derivation of the limiting ghost effect system.

\section{Kinetic Theory of Binary Gaseous Mixture: Background}

This section deals with the review of the main properties and results for the Boltzmann equations system (1.1). The aim of this section is to summarize the basic tools necessary for the derivation of the macroscopic fluid dynamics equations that will be performed in the next section. 
The first step is to write the Boltzmann equations system (1.1) in compact form. Let $[\cdot, \cdot]$ denote a column vector so that $F(t, x, v)=\left[F^{\mathrm{L}}, F^{\mathrm{H}}\right]$, writing the collision operator as follows:

$$
\mathscr{C}(F):=\left(\begin{array}{l}
Q\left(F^{L}, F^{L}\right)+Q\left(F^{\mathrm{H}}, F^{L}\right) \\
Q\left(F^{L}, F^{\mathrm{H}}\right)+Q\left(F^{\mathrm{H}}, F^{\mathrm{H}}\right)
\end{array}\right)
$$

the Boltzmann equations system (1.1) now reads:

$$
\partial_{t} F+v \cdot \nabla_{x} F=\mathscr{C}(F) .
$$

Moreover, for notational convenience, we set

$$
Q^{L} \equiv Q\left(F^{\mathrm{L}}, F^{\mathrm{L}}\right), \quad Q^{\mathrm{LH}} \equiv Q\left(F^{\mathrm{L}}, F^{\mathrm{H}}\right), \quad Q^{\mathrm{HL}} \equiv Q\left(F^{\mathrm{H}}, F^{\mathrm{L}}\right), \quad Q^{\mathrm{H}} \equiv Q\left(F^{\mathrm{H}}, F^{\mathrm{H}}\right) .
$$

Bearing all above in mind, the following main properties of the collision operator $Q$ are summarized (see, among others, papers [16], [15], [24], [2], [12] for further details):

(i) Mass conservation:

$$
\int_{\mathbb{R}^{3}} Q^{L} d v=0, \quad \int_{\mathbb{R}^{3}} Q^{\mathrm{LH}} d v=0, \quad \int_{\mathbb{R}^{3}} Q^{\mathrm{HL}} d v=0, \quad \int_{\mathbb{R}^{3}} Q^{\mathrm{H}} d v=0 .
$$

(ii) Momentum conservation:

$$
\int_{\mathbb{R}^{3}} Q^{L} m^{\mathrm{L}} v d v=0, \quad \int_{\mathbb{R}^{3}}\left(Q^{\mathrm{LH}} m^{\mathrm{L}} v+Q^{\mathrm{HL}} m^{\mathrm{H}} v\right) d v=0, \quad \int_{\mathbb{R}^{3}} Q^{\mathrm{H}} m^{\mathrm{H}} v d v=0 .
$$

(iii) Energy conservation:

$$
\int_{\mathbb{R}^{3}} Q^{L} m^{\mathrm{L}}|v|^{2} d v=0, \quad \int_{\mathbb{R}^{3}}\left(Q^{\mathrm{LH}} m^{\mathrm{L}}|v|^{2}+Q^{\mathrm{HL}} m^{\mathrm{H}}|v|^{2}\right) d v=0, \quad \int_{\mathbb{R}^{3}} Q^{\mathrm{H}} m^{\mathrm{H}}|v|^{2} d v=0 .
$$

(iv) Entropy inequalities: For each function $G=\left(G^{\mathrm{L}}, G^{\mathrm{H}}\right)^{T} \in \mathscr{C}_{0}^{\infty}\left(\mathbb{R}^{+} \times \mathbb{R}_{v}^{3}\right)$ we have:

$$
(\mathscr{C}(G), \log G)_{\left(L^{2}\left(\mathbb{R}^{3}\right)\right)^{2}}=\left(\mathscr{C}(G),\left(\begin{array}{l}
\log G^{L} \\
\log G^{\mathrm{H}}
\end{array}\right)\right)_{\left(L^{2}\left(\mathbb{R}^{3}\right)\right)^{2}} \leq 0,
$$

where $(\cdot, \cdot)$ denotes the inner product in $\left(L^{2}\left(\mathbb{R}^{3}\right)\right)^{2}$.

(v) Local thermal equilibria: The distribution function which maximizes the entropy (equilibrium distribution) has the form of a locally Maxwellian distribution, namely $(\mathscr{C}(G), \log G)=0$ if and only if $G$ reads:

$$
G=\left(\begin{array}{l}
n^{\mathrm{L}}\left(\frac{m^{\mathrm{L}}}{2 \pi \theta}\right)^{3 / 2} \exp \left(-m^{\mathrm{L}} \frac{|v-u|^{2}}{2 \theta}\right) \\
n^{\mathrm{H}}\left(\frac{m^{\mathrm{H}}}{2 \pi \theta}\right)^{3 / 2} \exp \left(-m^{\mathrm{H}} \frac{|v-u|^{2}}{2 \theta}\right)
\end{array}\right),
$$

where $u^{\mathrm{L}}=u^{\mathrm{H}}=u \in \mathbb{R}^{3}$ is the mean velocity, $\theta^{\mathrm{L}}=\theta^{\mathrm{H}}=\theta \in \mathbb{R}^{+}$is the local temperature and $n^{\alpha}=\int_{\mathbb{R}^{3}} G^{\alpha} d v \in \mathbb{R}^{+}$is the number density of the gas $\alpha$. Note that $n^{\alpha}>0$ guarantees the positivity of the distribution function and $\theta>0$ ensures the integrability with respect to $v$.

For simplicity we set:

$$
\begin{aligned}
& \mathcal{M}^{\alpha} \equiv \mathcal{M}_{\left[n^{\alpha}, u, \theta\right]}(v)=n^{\alpha}\left(\frac{m^{\alpha}}{2 \pi \theta}\right)^{3 / 2} \exp \left(-m^{\alpha} \frac{|v-u|^{2}}{2 \theta}\right), \quad \alpha \in\{\mathrm{L}, \mathrm{H}\} \\
& \mu^{\alpha}(v) \equiv \mathcal{M}^{\alpha} \equiv \mathcal{M}_{[1,0,1]}(v)(v)=\left(\frac{m^{\alpha}}{2 \pi}\right)^{3 / 2} \exp \left(-m^{\alpha} \frac{|v|^{2}}{2}\right), \quad \alpha \in\{\mathrm{L}, \mathrm{H}\}
\end{aligned}
$$


and

Therefore we have:

$$
\mu(v)=(2 \pi)^{-3 / 2} e^{-|v|^{2} / 2} .
$$

$$
Q^{\mathrm{L}}\left(\mathcal{M}^{\mathrm{L}}, \mathcal{M}^{\mathrm{L}}\right)+Q^{\mathrm{LH}}\left(\mathcal{M}^{\mathrm{L}}, \mathcal{M}^{\mathrm{H}}\right)=0, \quad \text { and } \quad Q^{\mathrm{HL}}\left(\mathcal{M}^{\mathrm{H}}, \mathcal{M}^{\mathrm{L}}\right)+Q^{\mathrm{H}}\left(\mathcal{M}^{\mathrm{H}}, \mathcal{M}^{\mathrm{H}}\right)=0 .
$$

The density $n^{\alpha}$, the mean velocity $u^{\alpha}=u$ and the temperature $\theta^{\alpha}=\theta$ are such that

$$
\int \mathcal{M}_{\left[n^{\alpha}, u, \theta\right]}(v)\left(\begin{array}{c}
1 \\
m^{\alpha} v \\
m^{\alpha}|v|^{2}
\end{array}\right) d v=\left(\begin{array}{c}
n^{\alpha} \\
m^{\alpha} n^{\alpha} u \\
m^{\alpha} n^{\alpha}|u|^{2}+3 \kappa \theta
\end{array}\right) .
$$

The quantities $m^{\alpha} n^{\alpha} u^{\alpha}$ and $\mathcal{W}_{\mathcal{M}}^{\alpha}:=\frac{1}{2} m^{\alpha} n^{\alpha}|u|^{2}+\frac{3}{2} \kappa \theta$ are the momentum and energy densities of the Maxwellian and $\kappa$ is the Boltzmann constant.

We further normalize the collision operator (1.2) as follows:

$$
Q\left(F^{\alpha}, F^{\ell}\right)=\int_{\mathbb{S}^{+} \times \mathbb{R}^{3}}\left|\left(v_{*}-v\right), \omega\right|\left[F^{\alpha}\left(v_{*}^{\prime}\right) F^{\ell}\left(v^{\prime}\right)-F^{\alpha}\left(v_{*}\right) F^{\ell}(v)\right] d \omega d v_{*} .
$$

Next, we define the standard perturbation $F^{\alpha}$ with respect to $\mu$ as follows:

$$
F^{\alpha}=\mu+f^{\alpha} \sqrt{\mu}, \quad \alpha \in\{\mathrm{L}, \mathrm{H}\} .
$$

We then study the normalized vector-valued binary gaseous mixture Boltzmann equations system for the perturbation

$$
f(t, x, v)=\left[f^{\mathrm{L}}(t, x, v), f^{\mathrm{H}}(t, x, v)\right],
$$

which now takes the form

$$
\left\{\partial_{t}+v \cdot \nabla_{x}\right\} f+\mathcal{L} f=\Gamma(f, f),
$$

where, for each given $g=\left[g^{\mathrm{L}}, g^{\mathrm{H}}\right]$, the linearized collision operator in (2.14) reads:

$$
\mathcal{L}=\left[\mathcal{L}^{\mathrm{L}}, \mathcal{L}^{\mathrm{H}}\right]:=[\mathbb{L} g, \mathbb{H} g]
$$

where

$$
-\mathcal{L} g=\mu^{-1 / 2}\left(\begin{array}{c}
2 Q^{\mathrm{LL}}\left(\mu, \mu^{\frac{1}{2}} g^{\mathrm{L}}\right)+Q^{\mathrm{HL}}\left(\mu, \mu^{\frac{1}{2}}\left(g^{\mathrm{L}}+g^{\mathrm{H}}\right)\right) \\
Q^{\mathrm{LH}}\left(\mu, \mu^{\frac{1}{2}}\left(g^{\mathrm{L}}+g^{\mathrm{H}}\right)\right)+2 Q^{\mathrm{HH}}\left(\mu, \mu^{\frac{1}{2}} g^{\mathrm{H}}\right)
\end{array}\right) .
$$

Simple calculations show that $\mathcal{L}$ can be decomposed in the standard way (see for instance [14]) as: $\mathcal{L} g=\nu(v) g-\mathcal{K} g$, where $\mathcal{K}$ is a compact integral operator on the space of square integrable functions, and the function $\nu(v)$, called collision frequency, is a smooth function growing at large velocities as the cross section that reads:

$$
\nu(v) \equiv \int_{\mathbb{R}^{3} \times \mathbb{S}^{2}}|v-u| \mu(u) d u d \omega .
$$

For $g$ and $h$ the nonlinear collision operator is

$$
\Gamma(g, h)=\mu^{-1 / 2}\left(\begin{array}{c}
Q\left(\mu^{\frac{1}{2}} g^{\mathrm{L}}, \mu^{\frac{1}{2}} h^{\mathrm{L}}\right)+Q^{\mathrm{HL}}\left(\mu^{\frac{1}{2}} g^{\mathrm{H}}, \mu^{\frac{1}{2}} h^{\mathrm{L}}\right) \\
Q^{\mathrm{LH}}\left(\mu^{\frac{1}{2}} g^{\mathrm{L}}, \mu^{\frac{1}{2}} h^{\mathrm{H}}\right)+Q^{\mathrm{HH}}\left(\mu^{\frac{1}{2}} g^{\mathrm{H}}, \mu^{\frac{1}{2}} h^{\mathrm{H}}\right)
\end{array}\right) .
$$

Let $L_{\mathcal{M}}^{2}$ be the associated Hilbert space endowed with the usual $L^{2}$ inner product

$$
\left\langle\left(\begin{array}{l}
f^{\mathrm{L}} \\
f^{\mathrm{H}}
\end{array}\right),\left(\begin{array}{l}
g^{\mathrm{L}} \\
g^{\mathrm{H}}
\end{array}\right)\right\rangle_{\mathcal{M}}=\int_{\mathbb{R}^{3}}\left(\mathcal{M}^{\mathrm{L}} f^{\mathrm{L}} g^{\mathrm{L}}+\mathcal{M}^{\mathrm{H}} f^{\mathrm{H}} g^{\mathrm{H}}\right) d v .
$$

The main properties of $\mathcal{L}$ are summarized in the following lemma (see Proposition 2.1, pp. 635-638, [1]):

Lemma 2.1. Assuming the hard-sphere interaction for the collision kernel, we have: 
1. $\mathcal{L}$ is the sum of a diagonal operator $f \mapsto \nu f$ and a compact operator $\mathcal{K}$. The domain of $\mathcal{L}$ is given by

$$
\mathcal{D}(\mathcal{L})=\left\{f:\left\|(1+|v|)^{\frac{1}{2}} f\right\|_{L_{\mathcal{M}}^{1}}<\infty\right\} .
$$

2. $\mathcal{L}$ is a nonnegative self-adjoint operator in $L_{\mathcal{M}}^{2}$ :

$$
\left\langle\mathcal{L}\left(\begin{array}{c}
f^{L} \\
f^{H}
\end{array}\right),\left(\begin{array}{c}
g^{L} \\
g^{H}
\end{array}\right)\right\rangle_{\mathcal{M}}=\left\langle\left(\begin{array}{l}
f^{L} \\
f^{H}
\end{array}\right), \mathcal{L}\left(\begin{array}{l}
g^{L} \\
g^{H}
\end{array}\right)\right\rangle_{\mathcal{M}}
$$

3. The kernel of $\mathcal{L}$ is a six-dimensional linear space:

$$
\text { where } \quad \mathfrak{N}:=\operatorname{ker}(\mathcal{L})=\operatorname{Span}\left\{\phi_{i}, \quad i=0, \ldots, 5\right\}
$$

$$
\phi_{0}=\left(\begin{array}{l}
1 \\
0
\end{array}\right), \phi_{1}=\left(\begin{array}{l}
0 \\
1
\end{array}\right), \phi_{i+1}=\left(\begin{array}{l}
m^{L} v_{i} \\
m^{H} v_{i}
\end{array}\right),(i=1,2,3), \quad \phi_{5}=\left(\begin{array}{l}
m^{L}|v|^{2} \\
m^{H}|v|^{2}
\end{array}\right) .
$$

4. Any function $f \in \mathcal{D}(\mathcal{L})$ can be written as $f=q_{f}+w_{f}$ with $q_{f} \in \operatorname{ker}(\mathcal{L})$ and $w \in(k e r(\mathcal{L}))^{\perp}$ and we have $\langle\mathbb{L} f, f\rangle_{\mathcal{M}} \geqslant \delta_{0}\left\|(1+|v|)^{\frac{1}{2}} w_{f}\right\|^{2}$.

We will consider the basis

and

$$
\left\{\begin{array}{l}
\psi_{0}(v)=\mu^{1 / 2}(v), \\
\psi_{i}(v)=v_{i} \mu^{1 / 2}(v), \quad(i=1,2,3) \\
\psi_{4}(v)=\frac{1}{2}\left(|v|^{2}-3\right) \mu^{1 / 2}(v),
\end{array}\right.
$$

We define $\mathbf{P}$ as the orthogonal projection $L^{2}\left(\mathbb{R}_{v}^{3}\right)$ to the null space $\mathfrak{N}$. With $(t, x, v)$ fixed, we then decompose any function $g(t, x, v)$ as

$$
g(t, x, v)=\mathbf{P} g(t, x, v)+(\mathbf{I}-\mathbf{P}) g(t, x, v) .
$$

Then $\mathbf{P} g$ is called the hydrodynamic part of $g$ and $(I-\mathbf{P}) g:=\mathbf{P}_{1}$ the microscopic part.

Let's now introduce the pseudo-inverse of the linear operator $\mathcal{L}$. The Fredholm alternative states that the equation

$$
\mathcal{L} g=\phi, \quad \phi \in \mathbf{R}(\mathcal{L}), \quad g \in \mathbf{R}(\mathcal{L})
$$

has a solution if and only if $\phi \in \mathfrak{N}^{\perp}$, in which case there is a unique solution in $\phi \in \mathfrak{N}^{\perp}$ denoted by $g=\mathcal{L}^{-1} \phi$. The Fredholm property of $\mathcal{L}$ implies that for every $p \in(1, \infty)$

$$
\mathcal{L}: L^{p}(\mu d v) \rightarrow L^{p}(\mu d v) \text { is bounded, }
$$

and that for every $\xi \in L^{p}(\mu d v)$ there exists a unique $\widehat{\xi} \in L^{p}(\mu d v)$ such that

$$
\mathcal{L} \widehat{\xi}=\mathbf{P}_{1} \xi, \quad \mathbf{P} \widehat{\xi}=0 .
$$

For every $\xi \in L^{p}(\mu d v)$ we define $\mathcal{L}^{-1} \xi=\widehat{\xi}$ where $\widehat{\xi}$ is determined above. This defines an operator $\mathcal{L}^{-1}$ such that

$$
\begin{aligned}
& \mathcal{L}^{-1}: L^{p}(\mu d v) \rightarrow L^{p}(\mu d v), \quad \text { is bounded, } \\
& \mathcal{L}^{-1} \mathcal{L}=\mathbf{P}_{1} \quad \text { over } L^{p}(\mu d v), \quad \mathcal{L} \mathcal{L}^{-1}=\mathbf{P}_{1} \quad \text { over } L^{p}(\mu d v)
\end{aligned}
$$

and $\mathfrak{N}\left(\mathcal{L}^{-1}\right)=\mathfrak{N}(\mathcal{L})$. The operator $\mathcal{L}^{-1}$ is the unique pseudo inverse of $\mathcal{L}$ with these properties.

In what follows we denote the weighted inner product of $h$ and $g$ in $L_{\mu}^{2}\left(\mathbb{R}^{3}\right)$ with respect to a given Maxwellian $\mu$ by:

$$
\langle h, g\rangle_{\mu} \equiv \int_{\mathbb{R}^{3}} h(v) g(v) \mu d v .
$$


2.1. Moments and conservation laws. Macroscopic quantities such as the number, momentum and energy densities are defined as moments of the distribution function with respect to the velocity. Specifically, the density $n^{\alpha}$, the mean velocity $u^{\alpha}\left(m^{\alpha} n^{\alpha} u^{\alpha}\right.$ is the $\alpha$ subsystem momentum density) and the energy density $\mathcal{W}^{\alpha}$ of the subsystem $\alpha$ are computed from the $\alpha$-subsystem distribution function $f^{\alpha}$ as follows:

$$
\left(\begin{array}{c}
n^{\alpha} \\
m^{\alpha} n^{\alpha} u^{\alpha} \\
\mathcal{W}^{\alpha}
\end{array}\right)=\int F^{\alpha}(v)\left(\begin{array}{c}
1 \\
m^{\alpha} v^{\alpha} \\
\frac{1}{2} m^{\alpha}|v|^{2}
\end{array}\right) d v
$$

The quantity $m^{\alpha} n^{\alpha} u^{\alpha}$ is the $\alpha$-species momentum density and $\mathcal{W}^{\alpha}$ is the energy density. Moreover the following fields can be defined:

$$
\begin{aligned}
& \left.\mathbf{c}=v-u^{\alpha} \quad \text { (random velocity with bulk velocity } u\right) \\
& q_{i}^{\alpha}=\frac{1}{2} \int_{\mathbb{R}^{3}} \mathbf{c}_{i}|c|^{2} F^{\alpha} d v \quad(i=1,2), \quad \text { (heat flow vector) } \\
& \left.p_{i j}^{\alpha}=\int_{\mathbb{R}^{3}} \mathbf{c}_{i} \mathbf{c}_{j} F^{\alpha} d v \quad(i, j=1,2), \quad \text { (stress tensor with components } p_{i j}^{\alpha}\right) .
\end{aligned}
$$

It is common to separate the drift motion (defined by the average velocity $u^{\alpha}$ ) and the random kinetic motion (defined by the velocity $\mathbf{c}$ ) in evaluating these integrals. By definition of $u^{\alpha}$, one has

where

$$
\int v \otimes v F^{\alpha} m^{\alpha} d v=m^{\alpha} n^{\alpha} u^{\alpha} \otimes u^{\alpha}+\mathbb{P}^{\alpha}
$$

and

$$
\mathbb{P}^{\alpha}:=\operatorname{trace}\left(p_{i j}\right)=\int\left(v-u^{\alpha}\right) \otimes\left(v-u^{\alpha}\right) m^{\alpha} F^{\alpha} d v, \quad(i, j=1,2),
$$

with

$$
\int \frac{1}{2} m^{\alpha}|v|^{2} v F^{\alpha} m^{\alpha} d v=\mathcal{W}^{\alpha} u^{\alpha}+\mathbb{P}^{\alpha} u^{\alpha}+\mathbb{Q}^{\alpha}
$$

$$
\mathbb{Q}^{\alpha}:=\int \frac{m^{\alpha}\left|v-u^{\alpha}\right|^{2}}{2}\left(v-u^{\alpha}\right) F^{\alpha} d v
$$

The internal energy per particle $e^{\alpha}$ and the temperature $\theta^{\alpha}$ of $\alpha$-species gas are defined as:

$$
e^{\alpha}=\frac{m^{\alpha}}{2 n^{\alpha}} \int_{\mathbb{R}^{3}}\left|v-u^{\alpha}\right|^{2} F^{\alpha} d v
$$

where, unless otherwise stated, the domain of integration is the whole space of $v$ (or of the variable of integration). We define the mass density $\varrho^{\alpha}$ by $\varrho^{\alpha}=m^{\alpha} n^{\alpha}$. We also define global quantities for the mixture: the counterparts of the mixture, i.e., the molecular number density $n$, mass density $\varrho$, mass average velocity $v$, pressure $p$ and temperature $\theta$, are expressed by a proper combination of the quantities above as

$$
n=\sum_{\alpha \in\{L, H\}} n^{\alpha}, \quad \varrho=\sum_{\alpha \in\{L, H\}} \varrho^{\alpha}, \quad E=n \kappa \theta=\sum_{\alpha \in\{L, H\}}\left(p^{\alpha}+\frac{1}{3}\left|u-v^{\alpha}\right|^{2}\right) .
$$

The following proposition holds true, see [12] for the proof.

Proposition 2.2. Let $F^{\alpha}=F^{\alpha}(t, x, v)$ be a solution of the Boltzmann mixture system (1.1) that is locally integrable and rapidly decaying in $v$ for each $(t, x)$. Then the mass, momentum 
and energy conservation laws hold:

$$
\begin{gathered}
\partial_{t} \int_{\mathbb{R}^{3}} F^{\alpha} v d v+\nabla_{x} \int_{\mathbb{R}^{3}} v F^{\alpha} d v=0 \\
\partial_{t} \int_{\mathbb{R}^{3}} m^{\alpha} v F^{\alpha} d v+\nabla_{x} \int_{\mathbb{R}^{3}} m^{\alpha} v \otimes v F^{\alpha} d v=\int_{\mathbb{R}^{3}} m^{\alpha} v Q\left(F^{\alpha}, F^{\ell}\right) d v \\
\partial_{t} \int_{\mathbb{R}^{3}} m^{\alpha} \frac{1}{2}|v|^{2} F^{\alpha} d v+\nabla_{x} \int_{\mathbb{R}^{3}} m^{\alpha} \frac{1}{2}|v| L^{2} v F^{\alpha} d v=\int_{\mathbb{R}^{3}} m^{\alpha} \frac{1}{2}|v|^{2} Q\left(F^{\alpha}, F^{\ell}\right) d v
\end{gathered}
$$

Bearing the previous notations for the thermodynamic fields in mind, these continuity equations are, for the $L$-subsystem:

$$
\begin{gathered}
\partial_{t} n^{\mathrm{L}}+\nabla_{x} \cdot\left(n^{\mathrm{L}} u\right)=0 \\
\partial_{t}\left(m^{\mathrm{L}} n^{\mathrm{L}} u\right)+\nabla_{x}\left(\int v \otimes v F^{\mathrm{L}} m^{\mathrm{L}} d v\right)=\mathcal{Q}^{\mathrm{LH}} \\
\partial_{t} \mathcal{W}^{\mathrm{L}}+\nabla_{x} \cdot\left(\int \frac{m^{\mathrm{L}}|v|^{2}}{2} v F^{\mathrm{L}} d v\right)=\mathscr{Q}^{\mathrm{LH}}
\end{gathered}
$$

and similarly for the subsystem $H$ :

$$
\begin{gathered}
\partial_{t} n^{\mathrm{H}}+\nabla_{x} \cdot\left(n^{\mathrm{H}} u\right)=0 \\
\partial_{t}\left(m^{\mathrm{H}} n^{\mathrm{H}} u\right)+\nabla_{x}\left(\int v \otimes v F^{\mathrm{H}} m^{\mathrm{H}} d v\right)=\mathcal{Q}^{\mathrm{HL}} \\
\partial_{t} \mathcal{W}^{\mathrm{H}}+\nabla_{x} \cdot\left(\int \frac{m^{\mathrm{H}}|v|^{2}}{2} v F^{\mathrm{H}} d v\right)=\mathscr{Q}^{\mathrm{HL}},
\end{gathered}
$$

where $\mathcal{Q}^{\alpha \ell}$ and $\mathscr{Q}^{\alpha \ell}$ are the momentum and energy transfer rates toward the subsystem $\alpha$ from the subsystem $\ell$ :

$$
\left(\begin{array}{c}
\mathcal{Q}^{\alpha \ell} \\
\mathscr{Q}^{\ell \alpha}
\end{array}\right)=\int Q^{\alpha \ell}\left(F^{\alpha}, F^{\ell}\right)(v)\left(\begin{array}{c}
m^{\alpha} v \\
\frac{1}{2} m^{\alpha}|v|^{2}
\end{array}\right) d v
$$

so that, because of the momentum and energy conservation properties of the unlike-collision operators (2.4) and (2.5), we have

$$
\mathcal{Q}^{\alpha \ell}+\mathcal{Q}^{\ell \alpha}=0, \quad \mathscr{Q}^{\alpha \ell}+\mathscr{Q}^{\ell \alpha}=0
$$

\section{Derivation of The macroscopic EQUATIOns}

This section is devoted to the formal derivation of the macroscopic equations for the density, momentum and temperature from the Boltzmann equations system for the binary mixture hard-sphere gas.

As already mentioned in the introduction, the main aim of this paper is the asymptotic derivation of macroscopic equations. In particular the derivation of different hydrodynamical equations depends on the time and space scales employed. Specifically we consider the following rescaled Boltzmann mixture equations system

$$
\begin{aligned}
\varepsilon^{\gamma} \partial_{t} F_{\varepsilon}^{\mathrm{H}}+v \cdot \nabla_{x} F_{\varepsilon}^{\mathrm{H}} & =\varepsilon^{q} Q\left(F_{\varepsilon}^{\mathrm{H}}, F_{\varepsilon}^{\mathrm{L}}\right)+\frac{1}{\varepsilon} Q\left(F_{\varepsilon}^{\mathrm{H}}, F_{\varepsilon}^{\mathrm{H}}\right) \\
\varepsilon^{\gamma+1} \partial_{t} F_{\varepsilon}^{\mathrm{L}}+v \cdot \nabla_{x} F_{\varepsilon}^{\mathrm{L}} & =\frac{1}{\varepsilon} Q\left(F_{\varepsilon}^{\mathrm{L}}, F_{\varepsilon}^{\mathrm{L}}\right)+\varepsilon^{q+1} Q\left(F_{\varepsilon}^{\mathrm{L}}, F_{\varepsilon}^{\mathrm{H}}\right),
\end{aligned}
$$

where $F_{\epsilon}^{\alpha}(t, x, v)=F^{\alpha}\left(\frac{t}{\varepsilon^{\gamma}}, \frac{x}{\epsilon}, v\right), q>1$ is a real parameter, $\epsilon>0$ is a real parameter that can be interpreted as the Knudsen number or the mean-free path between two intermolecular collisions, and $\gamma \geqslant 0$ is the strength parameter of the scaling. It is worth noting that the time scaling, which is related to the Strouhal number (ratio of the oscillation frequency to 
the bulk velocity), is introduced to suppress, when $\gamma \neq 0$, the acoustic modes varying in a faster timescale than rotational modes of the fluid.

In the hydrodynamic regime, where the Knudsen number vanishes, it is expected to obtain a continuum limit and thus reaches statistical equilibrium. Specifically the paper shows that, depending on the magnitude of the parameters which define the scaling, the macroscopic quantities (number density, mean velocity and local temperature) of the two subsystems are solution of the acoustic equation, the linear incompressible Euler equation and the incompressible Navier-Stokes equation. The derivation is formally tackled by the recent moment method proposed by Bardos et al. [3] and the results generalize the analysis performed in [4].

It is worth stressing that we did not try to get all possible scalings. In particular, one can easily derive simplified models to those we have here [4].

It is worth stressing that for the derivation of the rescaled equations system we can think the solutions of (3.1a)-(3.1b) as scaled solutions of (1.1a)-(1.1b), that is, if $F^{\alpha}(t, x, v)$ solves the Boltzmann mixture equations (1.1a)-(1.1b) then the parabolic scaling $F_{\epsilon}^{\alpha}(t, x, v)=$ $F^{\alpha}\left(\frac{t}{\varepsilon^{\gamma}}, \frac{x}{\epsilon}, v\right)$ generates a solution of the dimensionless Boltzmann mixture equations system (1.1a)-(1.1b) (see for instance [3], [20]).

In the hydrodynamic regime where the Knudsen number vanishes $\epsilon \rightarrow 0$, it is clear that the right-hand side of (3.1a) would become singular. The only possibility to avoid this singularity is that

$$
\lim _{\varepsilon \rightarrow 0} Q\left(F_{\varepsilon}^{\mathrm{H}}, F_{\varepsilon}^{\mathrm{H}}\right)=0
$$

Then, by (2.7), the limit must be a Maxwellian, namely,

$$
F_{0}^{\mathrm{H}}=\mathcal{M}\left[n^{\mathrm{H}}, u, \theta\right],
$$

for some $\left(n^{\mathrm{H}}, u, \theta\right)$ which can be function of $t$ and $x$ (similarly for the subsystem L).

If $F_{\varepsilon}^{\mathrm{L}}$ and $F_{\varepsilon}^{\mathrm{H}}$ solve the binary gas mixture Boltzmann equations (3.1b) and (3.1a) then $F_{\varepsilon}^{\mathrm{L}}$ satisfies the local conservation laws of mass, momentum, and energy:

$\varepsilon^{\gamma+1} \partial_{t} \int_{\mathbb{R}^{3}} F_{\varepsilon}^{\mathrm{L}}\left(\begin{array}{c}1 \\ v m^{\mathrm{L}} \\ \frac{1}{2}|v|^{2} m^{\mathrm{L}}\end{array}\right) d v+\operatorname{div}_{x} \int_{\mathbb{R}^{3}} F_{\varepsilon}^{\mathrm{L}}\left(\begin{array}{c}v \\ v \otimes v m^{\mathrm{L}} \\ v \frac{1}{2}|v|^{2} m^{\mathrm{L}}\end{array}\right) d v=\varepsilon^{q+1} m^{\mathrm{L}}\left(\begin{array}{c}0 \\ \mathcal{Q}\left(F_{\varepsilon}^{\mathrm{L}}, F_{\varepsilon}^{\mathrm{H}}\right) \\ \mathscr{Q}\left(F_{\varepsilon}^{\mathrm{L}}, F_{\varepsilon}^{\mathrm{H}}\right)\end{array}\right)$

and for $F_{\varepsilon}^{\mathrm{H}}$ one has:

$$
\varepsilon^{\gamma} \partial_{t} \int_{\mathbb{R}^{3}} F_{\varepsilon}^{\mathrm{H}}\left(\begin{array}{c}
1 \\
v m^{\mathrm{H}} \\
\frac{1}{2}|v|^{2} m^{\mathrm{H}}
\end{array}\right) d v+\operatorname{div}_{x} \int_{\mathbb{R}^{3}} F_{\varepsilon}^{\mathrm{H}}\left(\begin{array}{c}
v \\
v \otimes v m^{\mathrm{H}} \\
v \frac{1}{2}|v|^{2} m^{\mathrm{L}}
\end{array}\right) d v=\varepsilon^{q} m^{\mathrm{H}}\left(\begin{array}{c}
0 \\
\mathcal{Q}\left(F_{\varepsilon}^{\mathrm{H}}, F_{\varepsilon}^{\mathrm{L}}\right) \\
\mathscr{Q}\left(F_{\varepsilon}^{\mathrm{H}}, F_{\varepsilon}^{\mathrm{L}}\right)
\end{array}\right) .
$$

Since the solutions of the asymptotic equations are not guaranteed to exist or to be regular, our proof is only formal. We assume that for each $\varepsilon>0, F_{\varepsilon}^{\alpha}$ is a solution of (3.1b) and (3.1a) that satisfies the local conservation laws of mass, momentum, and energy, as well as the local entropy relation. Assume that

as well as

$$
F_{\varepsilon}^{\alpha} \rightarrow F^{\alpha} \text { a.e }
$$

$$
\int_{\mathbb{R}^{3}} F_{\varepsilon}^{\alpha} d v \rightarrow \int_{\mathbb{R}^{3}} F^{\alpha} d v, \quad \int_{\mathbb{R}^{3}} v F_{\varepsilon}^{\alpha} d v \rightarrow \int_{\mathbb{R}^{3}} v F^{\alpha} d v, \quad \int_{\mathbb{R}^{3}}|v|^{2} F_{\varepsilon}^{\alpha} d v \rightarrow \int_{\mathbb{R}^{3}}|v|^{2} F^{\alpha} d v
$$

in $\mathscr{C}\left(\mathbb{R}^{+} ; \mathscr{D}^{\prime}\left(\mathbb{R}^{3}\right)\right)$, while

$$
\left.\int_{\mathbb{R}^{3}} v \otimes v F_{\varepsilon}^{\alpha} d v \rightarrow \int_{\mathbb{R}^{3}} v \otimes v F^{\alpha} d v \quad \int_{\mathbb{R}^{3}} v|v|^{2} F_{\varepsilon}^{\alpha} d v \rightarrow \int_{\mathbb{R}^{3}} v|v|^{2} F^{\alpha} d v \mathscr{D}^{\prime}\left(\mathbb{R}^{3}\right)\right),
$$

in $\mathscr{C}\left(\mathbb{R}^{+} ; \mathscr{D}^{\prime}\left(\mathbb{R}^{3}\right)\right)$, as $\varepsilon \rightarrow 0$. 
The small Mach number (ratio of the bulk velocity to the sound speed) is realized if $F_{\varepsilon}^{\alpha}$ is close to an absolute Maxwellian. If one takes the standard Maxwellian, the distance from this absolute Maxwellian can be scaled in the unit of the Knudsen number $\varepsilon$ as

$$
F_{\varepsilon}^{\alpha}(t, x, v)=\mu_{\alpha}+\varepsilon^{\beta} \mu_{\alpha}^{1 / 2}(v) g_{\varepsilon}^{\alpha}\left(t^{\prime}, x, v\right), \quad \alpha \in\{L, H\}
$$

where $\beta \geqslant 0$ is the strength parameter of the scaling. The distribution function $g^{\alpha}$ can be viewed as the microscopic response of the system to gradients of macroscopic variables.

Varying the magnitude of the two scalings, it yields different limits as $\varepsilon \rightarrow 0$. Thus, one can derive different fluid equations (and in particular incompressible models) depending on the chosen scaling.

Before going further into the analysis, a linearization of $F_{\varepsilon}^{\alpha}$ is required. We will look for the solution $F_{\varepsilon}^{\alpha}$ near $\mu$, that is, the solution having the form

$$
F_{\varepsilon}^{\mathrm{H}}=\mu+\varepsilon^{\beta} \mu^{\frac{1}{2}} g_{\varepsilon}^{\mathrm{H}}, \quad F_{\varepsilon}^{\mathrm{L}}=\mu+\varepsilon^{\mathbf{m}} \mu^{\frac{1}{2}} g_{\varepsilon}^{\mathrm{L}},
$$

with $g_{\varepsilon}^{\alpha}=O(1)$ as $\varepsilon \rightarrow 0$. Plugging the scaling (3.7) into the mixture system (3.1) to deduce the governing equation of the new unknown $g_{\varepsilon}^{\alpha}$, one finds:

$$
\begin{aligned}
\epsilon^{\gamma+1} \partial_{t} g_{\epsilon}^{L}+v \cdot \nabla_{x} g_{\epsilon}^{L}= & \frac{1}{\varepsilon} \mathbb{L}\left(g_{\epsilon}^{L}\right)+\varepsilon^{\mathbf{m}-\mathbf{1}} \Gamma\left(g_{\epsilon}^{L}\right)+\mu^{-\frac{1}{2}}\left\{\epsilon^{\beta+q-m+1} Q^{L H}\left(\mu, \mu^{-\frac{1}{2}} g_{\epsilon}^{H}\right)\right. \\
& \left.+\epsilon^{q+1} Q^{L H}\left(\mu^{-\frac{1}{2}} g_{\epsilon}^{L}, \mu\right)+\epsilon^{\beta+q+1} Q^{L H}\left(\mu^{\frac{1}{2}} g_{\varepsilon}^{\mathrm{L}}, \mu^{\frac{1}{2}} g_{\varepsilon}^{\mathrm{H}}\right)\right\} \\
\varepsilon^{\gamma} \partial_{t} F_{\varepsilon}^{\mathrm{H}}+v \cdot \nabla_{x} F_{\varepsilon}^{\mathrm{H}}= & \frac{1}{\varepsilon} \mathbb{H}\left(g_{\epsilon}^{H}\right)+\varepsilon^{\beta-1} \Gamma\left(g_{\epsilon}^{H}\right)+\mu^{-\frac{1}{2}}\left\{\varepsilon^{\mathbf{m}+\mathbf{q}-\beta} Q^{H L}\left(\mu, \mu^{\frac{1}{2}} g_{\varepsilon}^{\mathrm{L}}\right)\right. \\
& \left.+\varepsilon^{\mathbf{q}} Q^{L H}\left(\mu^{\frac{1}{2}} g_{\varepsilon}^{\mathrm{H}}, \mu\right)+\varepsilon^{\mathbf{m}+\mathbf{q}} Q^{H L}\left(\mu^{\frac{1}{2}} g_{\varepsilon}^{\mathrm{H}}, \mu^{\frac{1}{2}} g_{\varepsilon}^{\mathrm{L}}\right)\right\} .
\end{aligned}
$$

Here, $\varepsilon^{\gamma}$ allows us to choose the phenomenon we want to emphasis. For notational convenience, we set:

$$
\begin{aligned}
\mathcal{L}_{1}^{\mathrm{LH}}\left(g_{\epsilon}^{\mathrm{LH}}\right)=\mu^{-\frac{1}{2}} Q^{L H}\left(\mu, \mu^{-\frac{1}{2}} g_{\epsilon}^{H}\right), & \mathcal{L}_{2}^{\mathrm{LH}}\left(g_{\epsilon}^{\mathrm{LH}}\right)=\mu^{-\frac{1}{2}} Q^{L H}\left(\mu^{-\frac{1}{2}} g_{\epsilon}^{L}, \mu\right) \\
\mathcal{H}_{1}^{\mathrm{HL}}\left(g_{\epsilon}^{\mathrm{HL}}\right)=\mu^{-\frac{1}{2}} Q^{H L}\left(\mu, \mu^{\frac{1}{2}} g_{\varepsilon}^{\mathrm{L}}\right) & \mathcal{H}_{2}^{\mathrm{HL}}\left(g_{\epsilon}^{\mathrm{HL}}\right)=\mu^{-\frac{1}{2}} Q^{L H}\left(\mu^{\frac{1}{2}} g_{\varepsilon}^{\mathrm{H}}, \mu\right) \\
\Gamma_{1}^{\mathrm{LH}}\left(g_{\epsilon}^{\mathrm{LH}}\right)=\mu^{-\frac{1}{2}} Q^{L H}\left(\mu^{\frac{1}{2}} g_{\varepsilon}^{\mathrm{L}}, \mu^{\frac{1}{2}} g_{\varepsilon}^{\mathrm{H}}\right), & \Gamma_{2}^{\mathrm{HL}}\left(g_{\epsilon}^{\mathrm{HL}}\right)=\mu^{-\frac{1}{2}} Q^{H L}\left(\mu^{\frac{1}{2}} g_{\varepsilon}^{\mathrm{H}}, \mu^{\frac{1}{2}} g_{\varepsilon}^{\mathrm{L}}\right) .
\end{aligned}
$$

By varying $\varepsilon, \beta$ we can formally derive the systems of the fluids dynamics.

The main result of the present paper is to perform the asymptotic limit when $\gamma, \beta>0$, $m>1$. Specifically, the evolution of $\left(n^{\mathrm{H}}, u, \theta\right)$ is governed by the acoustic system for $F^{\mathrm{H}}$ and Incompressible Navier-Stokes equations for $F^{\mathrm{L}}$.

We state the main result of this article.

Theorem 3.1. Let $F_{\varepsilon}^{L}$ and $F_{\varepsilon}^{H}$ be a family of distribution solutions to the scaled mixture systems (3.1b)-(3.1a) that satisfies the local conservation laws of mass, momentum, and energy. Moreover, for $\alpha, \beta \in\{L, H\}$, assume that:

$$
g_{\varepsilon}^{\alpha}=\frac{F_{\varepsilon}^{\alpha}-\mu}{\varepsilon \sqrt{\mu}} \rightarrow f^{\alpha} \quad \text { in the sense of distributions }
$$

and

$$
\varepsilon Q^{\alpha \beta}\left(g_{\varepsilon}^{\alpha}, g_{\varepsilon}^{\beta}\right) \rightarrow 0 \quad \text { in the sense of distributions }
$$


C. BIANCA AND C. DOGBE

$\begin{array}{cl}\int_{\mathbb{R}^{3}} g_{\varepsilon}^{\alpha} \sqrt{\mu} d v \rightarrow \int_{\mathbb{R}^{3}} g^{\alpha} \sqrt{\mu} d v, \quad \int_{\mathbb{R}^{3}} v g_{\varepsilon}^{\alpha} \sqrt{\mu} d v \rightarrow \int_{\mathbb{R}^{3}} v g^{\alpha} \sqrt{\mu} & \text { in } \mathscr{C}\left(\mathbb{R}^{+} ; \mathcal{D}\left(\mathbb{R}^{3}\right)\right), \\ \int_{\mathbb{R}^{3}} v \otimes v g_{\varepsilon}^{\alpha} \sqrt{\mu} d v \rightarrow \int_{\mathbb{R}^{3}} v \otimes v g^{\alpha} \sqrt{\mu} d v, \quad \int_{\mathbb{R}^{3}} v g_{\varepsilon}^{\alpha} \sqrt{\mu} d v \rightarrow \int_{\mathbb{R}^{3}} v g^{\alpha} \sqrt{\mu} d v & \text { in } \mathscr{C}\left(\mathbb{R}^{+} ; \mathcal{D}\left(\mathbb{R}^{3}\right)\right), \\ \int_{\mathbb{R}^{3}}|v|^{2} g_{\varepsilon}^{\alpha} \sqrt{\mu} d v \rightarrow \int_{\mathbb{R}^{3}}|v|^{2} g^{\alpha} \sqrt{\mu} d v, \quad \int_{\mathbb{R}^{3}} v|v|^{2} g_{\varepsilon}^{\alpha} \sqrt{\mu} d v \rightarrow \int_{\mathbb{R}^{3}} v|v|^{2} g^{\alpha} \sqrt{\mu} d v & \text { in } \mathscr{C}\left(\mathbb{R}^{+} ; \mathcal{D}\left(\mathbb{R}^{3}\right)\right),\end{array}$

as $\varepsilon \rightarrow 0$. Then the asymptotic limit $f:=\left[f_{\varepsilon}^{L}, f_{\varepsilon}^{H}\right]^{T}$ reads:

$$
f(t, x, v)=n(t, x, v)+v \cdot u(t, x, v)+\theta(t, x, v) \frac{1}{2}\left(|v|^{2}-3\right) .
$$

\section{Furthermore}

- If $\gamma=0, \quad \beta>0,(u, \theta)$ is solution of the linear compressible equation (the so-called acoustic equation) for the subsystem $H$ and solution of the incompressible Navier-Stokes equation for the subsystem $L$ :

$$
\left\{\begin{array} { l } 
{ \partial _ { t } n ^ { H } + \operatorname { d i v } u = 0 } \\
{ \partial _ { t } u + \nabla _ { x } ( n ^ { H } + \theta ) = 0 } \\
{ \partial _ { t } \theta + \frac { 2 } { 3 } \operatorname { d i v } u = 0 . }
\end{array} \quad \left\{\begin{array}{l}
\frac{\partial \tilde{u}}{\partial t}+\tilde{u} \cdot \nabla \tilde{u}+\nabla \tilde{p}^{L}=\nu \Delta \tilde{u} \\
\frac{\partial \tilde{\theta}}{\partial t}+\tilde{u} \cdot \nabla \tilde{\theta}=\kappa \Delta \tilde{\theta} .
\end{array}\right.\right.
$$

- If $\gamma>0$ and $\beta>0$, then $u$ satisfies the divergence conditions

while $n^{L}$ and $\theta$ satisfies

$$
\operatorname{div} u=0,
$$

$$
\nabla_{x}\left(n^{L}+\theta\right)=0 .
$$

- If $\mathbf{0}<\gamma=\beta<1$, then the subsystem $H$ is solution of the Incompressible Euler equation:

$$
\left\{\begin{array}{l}
\frac{\partial u}{\partial t}+u \cdot \nabla u+\nabla p^{H}=0 \\
\frac{\partial \theta}{\partial t}+u \cdot \nabla \theta=0,
\end{array}\right.
$$

and the subsystem $L$ is solution of the incompressible Navier-Stokes equation.

\section{Proof of Theorem 3.1}

In this subsection, we study the group which governs the acoustic waves and then we will pass to the limit in the equation of conservation of the momentum projected on the space of divergence-free vector fields. We apply the strategy of Bardos et al. [3] to derive the limiting equations.

- The Case $\gamma=0, \quad \beta>0$.

- For the subsystem $\mathrm{H}$, we get the acoustic system from the Boltzmann equation. We write $(3.9)$ as

$$
\begin{gathered}
\varepsilon\left(\frac{\partial g_{\varepsilon}^{\mathrm{H}}}{\partial t}+v \cdot \nabla_{x} g_{\varepsilon}^{\mathrm{H}}\right)=\mathbb{H}\left(g_{\epsilon}^{\mathrm{H}}\right)+\varepsilon^{\beta+1} \Gamma\left(g_{\epsilon}^{\mathrm{H}}\right)+\varepsilon^{m+q-\beta+1} \mathcal{H}_{1}^{\mathrm{HL}}\left(g_{\epsilon}^{\mathrm{HL}}\right)+\varepsilon^{q+1} \mathcal{H}_{2}^{\mathrm{HL}}\left(g_{\epsilon}^{\mathrm{HL}}\right) \\
+\varepsilon^{m+q+1} \Gamma_{2}^{\mathrm{HL}}\left(g_{\epsilon}^{\mathrm{HL}}\right)
\end{gathered}
$$


By letting $\varepsilon \rightarrow 0$ in (4.1), one finds that $\mathbb{H}\left(g_{0}^{\mathrm{H}}\right)=0$. Hence $g(t, x, \cdot)$ takes values in $\operatorname{Null}(\mathbb{H})$, the null space of $\mathbb{H}$. Because $g^{\mathrm{H}}$ is assumed to belong to $\mathbb{L}^{\infty}\left(d t ; L^{2}(\mu d v d x)\right)$, we conclude that the limit $g_{0}^{\mathrm{H}}$ has the form

$$
g_{0}^{\mathrm{H}}=\left\{n^{\mathrm{H}}+v \cdot u+\left(\frac{v^{2}}{2}-\frac{3}{2}\right) \theta\right\} \sqrt{\mu},
$$

where $n^{\mathrm{H}}, u$ and $\theta$ are functions of $t, x$. In order to determine the dynamics of $n^{\mathrm{H}}, u, \theta$, we project (3.9) onto $\left\{\mu^{1 / 2}, v \mu^{1 / 2},\left(\frac{v^{2}}{2}-\frac{3}{2}\right) \mu^{1 / 2}\right\}$. The inner product of (3.9) with $\psi_{j}$ gives $(\gamma=0)$ :

$$
\begin{gathered}
\partial_{t} \int_{\mathbb{R}^{3}} \mu^{1 / 2} g_{\varepsilon}^{\mathrm{H}} d v+\nabla_{x} \cdot \int_{\mathbb{R}^{3}} v \mu^{1 / 2} g_{\varepsilon}^{\mathrm{H}}=0 \\
\partial_{t} \int_{\mathbb{R}^{3}} v \mu^{1 / 2} g_{\varepsilon}^{\mathrm{H}} d v+\nabla_{x} \cdot \int_{\mathbb{R}^{3}} v \otimes v \mu^{1 / 2} g_{\varepsilon}^{\mathrm{H}} d v \\
\quad=\int_{\mathbb{R}^{3}} v \mu^{1 / 2}\left[\varepsilon^{m+q-\beta} \mathcal{H}_{1}^{\mathrm{HL}}\left(g_{\epsilon}^{\mathrm{HL}}\right)+\varepsilon^{q} \mathcal{H}_{2}^{\mathrm{HL}}\left(g_{\epsilon}^{\mathrm{HL}}\right)+\varepsilon^{m+q} \Gamma_{2}^{\mathrm{HL}}\left(g_{\epsilon}^{\mathrm{HL}}\right)\right] d v \\
\partial_{t} \int_{\mathbb{R}^{3}}\left(\frac{v^{2}}{2}-\frac{3}{2}\right) \mu^{1 / 2} g_{\varepsilon}^{\mathrm{H}} d v+\nabla_{x} \cdot \int_{\mathbb{R}^{3}} v\left(\frac{v^{2}}{2}-\frac{3}{2}\right) \mu^{1 / 2} g_{\varepsilon}^{\mathrm{H}} d v \\
=\int_{\mathbb{R}^{3}}\left(\frac{v^{2}}{2}-\frac{3}{2}\right) \mu^{1 / 2}\left[\varepsilon^{m+q-\beta} \mathcal{H}_{1}^{\mathrm{HL}}\left(g_{\epsilon}^{\mathrm{HL}}\right)+\varepsilon^{q} \mathcal{H}_{2}^{\mathrm{HL}}\left(g_{\epsilon}^{\mathrm{HL}}\right)+\varepsilon^{m+q} \Gamma_{2}^{\mathrm{HL}}\left(g_{\epsilon}^{\mathrm{HL}}\right)\right] d v .
\end{gathered}
$$

Next, passing to the limit in both sides of this equality, we arrive at

$$
\begin{gathered}
\partial_{t} \int_{\mathbb{R}^{3}} \mu^{1 / 2} g^{\mathrm{H}} d v+\nabla_{x} \cdot \int_{\mathbb{R}^{3}} v \mu^{1 / 2} g^{\mathrm{H}}=0 \\
\partial_{t} \int_{\mathbb{R}^{3}} v \mu^{1 / 2} g^{\mathrm{H}} d v+\nabla_{x} \cdot \int_{\mathbb{R}^{3}} v \otimes v \mu^{1 / 2} g^{\mathrm{H}} d v=0 \\
\partial_{t} \int_{\mathbb{R}^{3}}\left(\frac{v^{2}}{2}-\frac{3}{2}\right) \mu^{1 / 2} g^{\mathrm{H}} d v+\nabla_{x} \cdot \int_{\mathbb{R}^{3}} v\left(\frac{v^{2}}{2}-\frac{3}{2}\right) \mu^{1 / 2} g^{\mathrm{H}} d v=0
\end{gathered}
$$

Since $g^{\mathrm{H}}=\mathbf{P} g^{\mathrm{H}}$, the fluid variables associated with the fluctuation of the number density $g_{\varepsilon}$ is defined by:

$$
n_{\varepsilon}^{\mathrm{H}}=\left\langle g_{\varepsilon}^{\mathrm{H}}\right\rangle, \quad u_{\varepsilon}=\left\langle v g_{\varepsilon}^{\mathrm{H}}\right\rangle, \quad \theta_{\varepsilon}=\left\langle\left(\frac{v^{2}}{2}-\frac{3}{2}\right) g_{\varepsilon}^{\mathrm{H}}\right\rangle, \quad \text { with } \quad\langle\zeta\rangle=\int \zeta(v) \mu^{1 / 2}(v) d v .
$$

Therefore, in the limit one has

$$
\nabla_{x} \cdot \int_{\mathbb{R}^{3}} v \otimes v \mu^{1 / 2} g^{\mathrm{H}} d v=\nabla_{x} \cdot\left\langle v^{\otimes 2}\left(n^{\mathrm{H}}+v \cdot u+\left(\frac{v^{2}}{2}-\frac{3}{2}\right) \theta\right)\right\rangle .
$$

Observe that by our normalization, it follows that

$$
\begin{aligned}
& \langle 1, \mu\rangle=1, \quad\left\langle\left|v_{j}\right|^{2}, \mu\right\rangle=1, \quad\left\langle|v|^{2}, \mu\right\rangle=3 \\
& \left\langle\left|v_{j}\right|^{2}\left|v_{m}\right|^{2}, \mu\right\rangle=1, \quad j \neq m \\
& \left\langle|v|^{4}, \mu\right\rangle=15, \quad\left\langle\left|v_{j}\right|^{4}, \mu\right\rangle=3, \quad\left\langle|v|^{2}\left|v_{j}\right|^{2}, \mu\right\rangle=5
\end{aligned}
$$

and in view of the identity

$$
\left\langle v^{\otimes 2}\right\rangle=\left\langle v^{\otimes 2} \frac{1}{2}\left(|v|^{2}-3\right)\right\rangle=\left\langle\frac{1}{6}|v|^{2}\left(|v|^{2}-3\right)\right\rangle I=I,
$$

substituting the explicit formula for $g^{\mathrm{H}}$ in the left-hand side of the identity above leads to:

$$
\nabla_{x} \cdot\left\langle v \otimes v n^{\mathrm{H}}\right\rangle=\nabla_{x} \cdot\left(n^{\mathrm{H}} I\right)=\nabla_{x} n^{\mathrm{H}}
$$


the second term of (4.5) vanishes by the oddness of the integrand, while

$$
\nabla_{x} \cdot\left\langle v \otimes v\left(\frac{1}{2}|v|^{2}-\frac{3}{2}\right) \theta\right\rangle=\nabla_{x} \cdot\left\langle v \otimes v\left(\frac{1}{2}|v|^{2}-\frac{3}{2}\right)\right\rangle \theta=\nabla_{x} \cdot(\theta I)=\nabla_{x} \theta,
$$

since

Therefore,

$$
\left\langle v_{i} v_{j} \frac{1}{2}|v|^{2}\right\rangle=\frac{5}{2} \delta_{i j}
$$

$$
\nabla_{x}\left(n^{\mathrm{H}}+\theta\right)=0 .
$$

This result is known as the Boussinesq relation. It says that the fluctuations in the gradient of the density balance those in the gradient of the temperature.

Furthermore

$$
\left.\langle v\rangle=0, \quad\left\langle v^{3}\right\rangle=0, \quad\left\langle v^{\otimes 2}\left(\frac{v^{2}}{2}-\frac{3}{2}\right) \theta\right)\right\rangle=\theta .
$$

We conclude that $\left(n^{\mathrm{H}}, u, \theta\right)$ is solution of the following system

$$
\left\{\begin{array}{l}
\partial_{t} n^{\mathrm{H}}+\operatorname{div} u=0 \\
\partial_{t} u+\nabla_{x}\left(n^{\mathrm{H}}+\theta\right)=0 \\
\partial_{t} \theta+\frac{2}{3} \operatorname{div} u=0 .
\end{array}\right.
$$

- For the subsystem L, we are concerned with the equation

$$
\left\{\begin{array}{l}
\varepsilon \partial_{t} F_{\varepsilon}^{\mathrm{L}}+v \cdot \nabla_{x} F_{\varepsilon}^{\mathrm{L}}=\frac{1}{\varepsilon} Q\left(F_{\varepsilon}^{\mathrm{L}}, F_{\varepsilon}^{\mathrm{L}}\right)+\varepsilon^{q}+1 Q^{\mathrm{LH}}\left(F_{\varepsilon}^{\mathrm{L}}, F_{\varepsilon}^{\mathrm{H}}\right) \\
F_{\varepsilon}^{\mathrm{L}}=\mu+\varepsilon^{m} \mu^{\frac{1}{2}} g_{\varepsilon}^{\mathrm{L}} .
\end{array}\right.
$$

We get the incompressible Navier-Stokes equations. Indeed, the associated fluid dynamic variables, mass density $n_{\varepsilon}^{\mathrm{L}}$, bulk velocity $u_{\varepsilon}^{\mathrm{L}}$ and temperature $\theta_{\varepsilon}$ are expressed in terms of their fluctuations as

$$
n_{\varepsilon}^{\mathrm{L}}=1+\varepsilon \tilde{n}_{\varepsilon}^{\mathrm{L}}, \quad u_{\varepsilon}^{\mathrm{L}}=\varepsilon \tilde{u}_{\varepsilon}, \quad \theta_{\varepsilon}=1+\varepsilon \tilde{\theta}_{\varepsilon}^{\mathrm{L}} .
$$

Averaging the equation for the species L over the velocity domain yields

$\varepsilon \partial_{t}\left\langle g_{\epsilon}^{L}\right\rangle+v \cdot \nabla_{x}\left\langle g_{\epsilon}^{L}\right\rangle=\epsilon^{\beta+q-m+1}\left\langle\mathcal{L}_{1}^{\mathrm{LH}}\left(g_{\epsilon}^{\mathrm{LH}}\right)\right\rangle+\epsilon^{q+1}\left\langle\mathcal{L}_{2}^{\mathrm{LH}}\left(g_{\epsilon}^{\mathrm{LH}}\right)\right\rangle+\varepsilon^{\beta+q+1}\left\langle\Gamma_{1}^{\mathrm{LH}}\left(g_{\epsilon}^{\mathrm{LH}}\right)\right\rangle$.

According to our weak notion of solution for Boltzmann equation, this means that, for any smooth function $\phi(x)$ and any $t_{1}, t_{2}>0$,

$$
\begin{aligned}
& \varepsilon \int_{t_{1}}^{t_{2}} \frac{d}{d t} \int \phi(x)\left\langle g_{\varepsilon}^{\mathrm{L}}\right\rangle d x d t-\int_{t_{1}}^{t_{2}} \int \nabla_{x} \phi(x) \cdot\left\langle v g_{\varepsilon}^{\mathrm{L}}\right\rangle d x d t \\
= & \epsilon^{\beta+q-m+1} \int_{t_{1}}^{t_{2}} \int\left\langle\mathcal{L}_{1}^{\mathrm{LH}}\left(g_{\epsilon}^{\mathrm{LH}}\right), \phi(x)\right\rangle d x d t+\epsilon^{q+1} \int_{t_{1}}^{t_{2}} \int\left\langle\mathcal{L}_{2}^{\mathrm{LH}}\left(g_{\epsilon}^{\mathrm{LH}}\right), \phi(x)\right\rangle d x d t \\
& \left.+\epsilon^{\beta+q+1} \int_{t_{1}}^{t_{2}} \int\left\langle\Gamma_{1}^{\mathrm{LH}}\left(g_{\epsilon}^{\mathrm{LH}}\right), \mu^{\frac{1}{2}} g_{\varepsilon}^{\mathrm{H}}\right), \phi(x)\right\rangle d x d t
\end{aligned}
$$

or

$$
\begin{aligned}
& \varepsilon \int \phi(x)\left(\left\langle g_{\varepsilon}^{\mathrm{L}}\left(t_{2}\right)\right\rangle-\left\langle g_{\varepsilon}^{\mathrm{L}}\left(t_{1}\right)\right\rangle\right) d x-\int_{t_{1}}^{t_{2}} \int \nabla_{x} \phi(x) \cdot\left\langle v g_{\varepsilon}^{\mathrm{L}}\right\rangle d x d t \\
= & \epsilon^{\beta+q-m+1} \int_{t_{1}}^{t_{2}} \int\left\langle\mathcal{L}_{1}^{\mathrm{LH}}\left(g_{\epsilon}^{\mathrm{LH}}\right), \phi(x)\right\rangle d x d t+\epsilon^{q+1} \int_{t_{1}}^{t_{2}} \int\left\langle\mathcal{L}_{2}^{\mathrm{LH}}\left(g_{\epsilon}^{\mathrm{LH}}\right), \phi(x)\right\rangle d x d t \\
& \left.+\epsilon^{\beta+q+1} \int_{t_{1}}^{t_{2}} \int\left\langle\Gamma_{1}^{\mathrm{LH}}\left(g_{\epsilon}^{\mathrm{LH}}\right), \mu^{\frac{1}{2}} g_{\varepsilon}^{\mathrm{H}}\right), \phi(x)\right\rangle d x d t .
\end{aligned}
$$


Taking the limit as $\epsilon$ goes to zero, the first term on the left-hand side and the two terms of the right-hand side vanish (since the integrand is uniformly bounded), while the second one converges to

$$
\int_{t_{1}}^{t_{2}} \int_{\mathbb{R}^{3}} \nabla_{x} \phi(x) \cdot\left\langle v g^{\mathrm{L}}\right\rangle d x d t
$$

Substituting the above relation in the expression for $g^{\mathrm{L}}$ and using the orthogonality of the basis elements of the Nullspace of the operator $\mathbb{L}$, we are left with

$$
\left.\int_{t_{1}}^{t_{2}} \int_{\mathbb{R}^{3}} \nabla_{x} \phi(x) \cdot u(t, x)\right\rangle d x d t
$$

that is,

$$
\nabla_{x} \phi(x) \cdot u(t, x)=0
$$

satisfied in the weak sense stated above. This equation states the incompressibility of the fluid-like limiting behavior of the Boltzmann flow, while $n^{\mathrm{L}}$ and $\theta$ satisfy the Boussinesq relation

$$
\nabla_{x}\left(n^{\mathrm{L}}+\theta\right)=0 .
$$

Under the assumption of the theorem, if $\beta>0$, then $\mathbb{L}\left(g_{\varepsilon}^{\mathrm{L}}\right)=0$. We denote this limits $g_{0}^{\mathrm{L}}$. Using the basis defined in $(2.20)$ we write $g_{0}^{\mathrm{L}}$ as

$$
g_{0}^{\mathrm{L}}=\eta^{\mathrm{L}} \psi_{0}+\sum_{i=1}^{3} u_{i} \psi_{i}+\theta \psi_{4},
$$

so that one writes

$$
g_{0}^{\mathrm{L}}=\eta^{\mathrm{L}} \Psi_{0}+u \Psi_{1},
$$

where

$$
\Psi_{0} \equiv\left(\psi_{4}-\psi_{0}\right)=\frac{1}{2}\left(|v|^{2}-5\right) \psi_{0}, \quad \Psi_{1} \equiv\left(\psi_{1}, \psi_{2}, \psi_{3}\right)=v \psi_{0} .
$$

Recall that

$$
A(v)=\left(A_{i j}\right)=\psi_{0}(v)\left(v \otimes v-\frac{1}{3}|v|^{2} \mathbf{I}\right), \quad B(v)=\left(B_{i}(v)\right)=\frac{1}{2} \psi_{0}(v)\left(|v|^{2}-5\right) v
$$

Observe that

$$
v \otimes \Psi_{1}=A(v)+\frac{1}{3}|v|^{2} \psi_{0} \mathbf{I}, \quad \Psi_{0} v=B(v),
$$

and

$$
\left\langle\Psi_{0}, \Psi_{0}\right\rangle=\frac{5}{2}, \quad\left\langle\Psi_{1}, \Psi_{1}\right\rangle=\mathbf{I}, \quad\left\langle\Psi_{0}, \Psi_{1}\right\rangle=0
$$

component wise. Taking the inner product of (3.8) with $\psi_{j}$ we obtain:

$$
\begin{aligned}
\varepsilon \partial_{t}\left\langle g_{\epsilon}^{L}, \psi_{j}\right\rangle+v \cdot \nabla_{x}\left\langle g_{\epsilon}^{L}, \psi_{j}\right\rangle= & \epsilon^{\beta+q-m+1}\left\langle\mathcal{L}_{1}^{\mathrm{LH}}\left(g_{\epsilon}^{\mathrm{LH}}\right), \psi_{j}\right\rangle+\epsilon^{q+1}\left\langle\mathcal{L}_{2}^{\mathrm{LH}}\left(g_{\epsilon}^{\mathrm{LH}}\right), \psi_{j}\right\rangle \\
& +\epsilon^{\beta+q+1}\left\langle\Gamma_{1}^{\mathrm{LH}}\left(g_{\epsilon}^{\mathrm{LH}}\right), \psi_{j}\right\rangle,
\end{aligned}
$$

$j=0, \ldots, 4$. We write $(4.20)$ in the form $(j=0$ and $j=1, \ldots, 4)$ :

$$
\begin{aligned}
& \left.\frac{\partial}{\partial t}\left\langle g_{\varepsilon}^{\mathrm{L}}, \Psi_{0}\right\rangle+\frac{1}{\varepsilon} \nabla_{x} \cdot\left\langle\left(v \otimes v-\frac{1}{3} \mathbf{I}\right) g_{\varepsilon}^{\mathrm{L}}\right)\right\rangle+\frac{1}{\varepsilon} \nabla_{x} \cdot\left\langle\frac{1}{3}|v|^{2} g_{\varepsilon}^{\mathrm{L}}\right\rangle \\
& \quad=\epsilon^{\beta+q-m+1}\left\langle\mathcal{L}_{1}^{\mathrm{LH}}\left(g_{\epsilon}^{\mathrm{LH}}\right), \Psi_{0}\right\rangle+\epsilon^{q+1}\left\langle\mathcal{L}_{2}^{\mathrm{LH}}\left(g_{\epsilon}^{\mathrm{LH}}\right), \Psi_{0}\right\rangle+\epsilon^{\beta+q+1}\left\langle\Gamma_{1}^{\mathrm{LH}}\left(g_{\epsilon}^{\mathrm{LH}}\right), \Psi_{0}\right\rangle, \\
& \left.\frac{\partial}{\partial t}\left\langle g_{\varepsilon}^{\mathrm{L}}, \Psi_{1}\right\rangle+\frac{1}{\varepsilon} \nabla_{x} \cdot\left\langle\left(\frac{1}{2} v|v|^{2}-5\right) g_{\varepsilon}^{\mathrm{L}}\right)\right\rangle \\
& \quad=\epsilon^{\beta+q-m+1}\left\langle\mathcal{L}_{1}^{\mathrm{LH}}\left(g_{\epsilon}^{\mathrm{LH}}\right), \Psi_{1}\right\rangle+\epsilon^{q+1}\left\langle\mathcal{L}_{2}^{\mathrm{LH}}\left(g_{\epsilon}^{\mathrm{LH}}\right), \Psi_{1}\right\rangle+\epsilon^{\beta+q+1}\left\langle\Gamma_{1}^{\mathrm{LH}}\left(g_{\epsilon}^{\mathrm{LH}}\right), \Psi_{1}\right\rangle,
\end{aligned}
$$

The first equation comes from the subtraction of equations (4.20) for $j=4$ and $j=0$ and the second equation from the multiplication of (4.20) by $v$. The term $p_{\varepsilon} \equiv \frac{1}{3 \varepsilon^{\gamma+1}}|v|^{2} g_{\varepsilon}^{\alpha}$ and therefore it vanishes when, according to our prescription, we integrate (4.21) against 
a divergence-free test function. Therefore, we are going to ignore this term in the computation below.

Passing into the limit in $(4.21)$, we get

$$
\begin{aligned}
& \partial_{t}\left\langle g_{\varepsilon}^{\mathrm{L}}, \Psi_{0}\right\rangle \rightarrow \partial_{t}\left\langle g_{0}^{\mathrm{L}}, \Psi_{0}\right\rangle=\partial_{t}\left(\theta \Psi_{0}+u \cdot \Psi_{1}, \Psi_{0}\right\rangle=\frac{5}{2} \partial_{t} \theta \\
& \partial_{t}\left\langle g_{\varepsilon}^{\mathrm{L}}, \Psi_{1}\right\rangle \rightarrow \partial_{t}\left\langle g_{0}^{\mathrm{L}}, \Psi_{1}\right\rangle=\partial_{t}\left(\theta \Psi_{0}+u \cdot \Psi_{1}, \Psi_{1}\right\rangle=\partial_{t} u .
\end{aligned}
$$

Next, observe that from (3.8) one has

$$
\begin{aligned}
\mathbb{L}\left(g_{\epsilon}^{L}\right)= & \epsilon^{2} \partial_{t} g_{\epsilon}^{L}+\varepsilon v \cdot \nabla_{x} g_{\epsilon}^{L}-\varepsilon^{m} \Gamma\left(g_{\epsilon}^{L}\right)+\epsilon^{\beta+q-m+2} \mathcal{L}_{1}^{\mathrm{LH}}\left(g_{\epsilon}^{\mathrm{LH}}\right)-\epsilon^{q+2} \mathcal{L}_{2}^{\mathrm{LH}}\left(g_{\epsilon}^{\mathrm{LH}}\right) \\
& -\varepsilon^{\beta+q+2} \Gamma_{1}^{\mathrm{LH}}\left(g_{\epsilon}^{\mathrm{LH}}\right) .
\end{aligned}
$$

To get a closed equation for $g_{\varepsilon}^{\mathrm{L}}$, we have to inverse the operator $\mathbb{L}$. Thus, for the species L, we have

$$
\begin{aligned}
\left.\frac{1}{\varepsilon}\left\langle\left(v \otimes v-\frac{1}{3} \mathbf{I}\right), g_{\varepsilon}^{\mathrm{L}}\right)\right\rangle= & \frac{1}{\varepsilon}\left\langle\mathbb{L}\left(g_{\varepsilon}^{\mathrm{L}}\right), \mathbb{L}^{-1} A\right\rangle \\
= & \frac{1}{\varepsilon}\left\langle\varepsilon^{2} \partial_{t} g_{\varepsilon}^{\mathrm{L}}, A^{\prime}\right\rangle+\frac{1}{\varepsilon}\left\langle\varepsilon v \cdot \nabla_{x} g_{\varepsilon}^{\mathrm{L}}, A^{\prime}\right\rangle+\frac{1}{\varepsilon}\left\langle-\varepsilon^{m} \Gamma\left(g_{\epsilon}^{L}\right), A^{\prime}\right\rangle \\
& +\frac{1}{\varepsilon}\left\langle-\varepsilon^{\beta+q+2} \Gamma_{1}^{\mathrm{LH}}\left(g_{\epsilon}^{\mathrm{LH}}\right), A^{\prime}\right\rangle+\frac{1}{\varepsilon}\left\langle-\epsilon^{\beta+q-m+2} \mathcal{L}_{1}^{\mathrm{LH}}\left(g_{\epsilon}^{\mathrm{LH}}\right), A^{\prime}\right\rangle \\
& +\frac{1}{\varepsilon}\left\langle-\epsilon^{q+2} \mathcal{L}_{2}^{\mathrm{LH}}\left(g_{\epsilon}^{\mathrm{LH}}\right), A^{\prime}\right\rangle,
\end{aligned}
$$

where we recall that $A^{\prime}=\mathbb{L}^{-1} A, \quad B^{\prime}=\mathbb{L}^{-1} B$. In other words

$$
\begin{aligned}
& \frac{1}{\varepsilon}\left\langle\mathbb{L}\left(g_{\varepsilon}^{\mathrm{L}}\right), \mathbb{L}^{-1} A\right\rangle= \varepsilon\left\langle\partial_{t} g_{\varepsilon}^{\mathrm{L}}, A^{\prime}\right\rangle+\left\langle v \cdot \nabla_{x} g_{\varepsilon}^{\mathrm{L}}, A^{\prime}\right\rangle-\varepsilon^{\beta+q+1}\left\langle\Gamma_{1}^{\mathrm{LH}}\left(g_{\epsilon}^{\mathrm{LH}}\right), A^{\prime}\right\rangle \\
&-\varepsilon^{m-1}\left\langle\Gamma\left(g_{\epsilon}^{L}\right), A^{\prime}\right\rangle-\epsilon^{\beta+q-m+1}\left\langle\mathcal{L}_{1}^{\mathrm{LH}}\left(g_{\epsilon}^{\mathrm{LH}}\right), A^{\prime}\right\rangle \\
&-\epsilon^{q+1}\left\langle\mathcal{L}_{2}^{\mathrm{LH}}\left(g_{\epsilon}^{\mathrm{LH}}\right), A^{\prime}\right\rangle .
\end{aligned}
$$

Similarly

$$
\begin{aligned}
& \frac{1}{\varepsilon}\left\langle\mathbb{L}\left(g_{\varepsilon}^{\mathrm{L}}\right), \mathbb{L}^{-1} B\right\rangle= \varepsilon\left\langle\partial_{t} g_{\varepsilon}^{\mathrm{L}}, B^{\prime}\right\rangle+\left\langle v \cdot \nabla_{x} g_{\varepsilon}^{\mathrm{L}}, B^{\prime}\right\rangle-\varepsilon^{\beta+q+1}\left\langle\Gamma_{1}^{\mathrm{LH}}\left(g_{\epsilon}^{\mathrm{LH}}\right), B^{\prime}\right\rangle \\
&-\varepsilon^{m-1}\left\langle\Gamma\left(g_{\epsilon}^{L}\right), B^{\prime}\right\rangle-\epsilon^{\beta+q-m+1}\left\langle\mathcal{L}_{1}^{\mathrm{LH}}\left(g_{\epsilon}^{\mathrm{LH}}\right), A^{\prime}\right\rangle \\
&-\epsilon^{q+1}\left\langle\mathcal{L}_{2}^{\mathrm{LH}}\left(g_{\epsilon}^{\mathrm{LH}}\right), B^{\prime}\right\rangle .
\end{aligned}
$$

Go to the limit $\varepsilon \rightarrow 0$. We must compute the limit of the term $\nabla\left\langle v \cdot \nabla_{x} g_{\varepsilon}^{\mathrm{L}}, A^{\prime}\right\rangle$ in $(4.25)$.

$$
\begin{aligned}
\nabla \cdot\left\langle v \otimes \nabla_{x} g_{0}^{\mathrm{L}}, A^{\prime}\right\rangle & =\nabla \cdot\left\langle v \cdot \nabla_{x}\left(\theta \Psi_{0}+u \cdot \Psi_{1}\right), A^{\prime}\right\rangle \\
& =\nabla \cdot\left\langle v \cdot \nabla_{x}\left(\theta \Psi_{0}\right), A^{\prime}\right\rangle+\nabla \cdot\left\langle v \cdot \nabla_{x}\left(u \cdot \Psi_{1}\right), A^{\prime}\right\rangle \\
& =\partial_{i} \partial_{j} \theta\left\langle A_{i j}, A^{\prime}\right\rangle+\partial_{i} \partial_{j} u_{k}^{\mathrm{L}}\left\langle B_{i}, A^{\prime}\right\rangle \\
& =-\partial_{i} \partial_{j} \theta\left(\mu \delta_{i j}\right)=-\mu \Delta \theta .
\end{aligned}
$$

Similarly, the term in (4.26) takes the form:

$$
\begin{aligned}
\nabla \cdot\left\langle v \cdot \nabla_{x} g_{0}^{\mathrm{L}}, B^{\prime}\right\rangle & =\nabla \cdot\left\langle v \cdot \nabla_{x}\left(\theta \Psi_{0}+u \cdot \Psi_{1}\right), B^{\prime}\right\rangle \\
& =\partial_{i} \partial_{j} \theta\left\langle A_{i j}, B^{\prime}\right\rangle+\partial_{i} \partial_{j} u_{m}\left\langle B_{i}, B^{\prime}\right\rangle \\
& =-\mu \Delta u_{k} .
\end{aligned}
$$

In conclusion, we get the incompressible Navier-Stokes equation, namely:

$$
\left\{\begin{array}{l}
\frac{\partial \tilde{u}}{\partial t}+\tilde{u} \cdot \nabla \tilde{u}+\nabla \tilde{p}^{\mathrm{H}}=\nu \Delta \tilde{u} \\
\frac{\partial \tilde{\theta}}{\partial t}+\tilde{u} \cdot \nabla \tilde{\theta}=\kappa \Delta \tilde{\theta} .
\end{array}\right.
$$


- The Case $\mathbf{0}<\gamma=\beta<1$. (Incompressible Euler equation).

For the species $\mathrm{H}$, we have

$$
\begin{aligned}
\frac{1}{\varepsilon^{\gamma}}\left\langle\mathbb{H}\left(g_{\varepsilon}^{H}\right), \mathbb{H}^{-1} A\right\rangle= & \frac{1}{\varepsilon^{\gamma}}\left\langle\varepsilon^{\gamma+1} \partial_{t} g_{\varepsilon}^{H}, A^{\prime}\right\rangle+\frac{1}{\varepsilon^{\gamma}}\left\langle\varepsilon v \cdot \nabla_{x} g_{\varepsilon}^{H}, A^{\prime}\right\rangle+\frac{1}{\varepsilon^{\gamma}}\left\langle-\varepsilon^{\beta-1} \Gamma\left(g_{\epsilon}^{H}\right), A^{\prime}\right\rangle \\
& +\frac{1}{\varepsilon^{\gamma}}\left\langle-\varepsilon^{m+q-\beta} \mathcal{H}_{1}^{\mathrm{HL}}\left(g_{\epsilon}^{\mathrm{HL}}\right), A^{\prime}\right\rangle+\frac{1}{\varepsilon^{\gamma}}\left\langle-\varepsilon^{q} \mathcal{H}_{2}^{\mathrm{HL}}\left(g_{\epsilon}^{\mathrm{HL}}\right), A^{\prime}\right\rangle \\
& +\frac{1}{\varepsilon^{\gamma}}\left\langle-\varepsilon^{m+q} \Gamma_{2}^{\mathrm{HL}}\left(g_{\epsilon}^{\mathrm{HL}}\right), A^{\prime}\right\rangle
\end{aligned}
$$

which we rewrite as

$$
\begin{aligned}
\frac{1}{\varepsilon^{\gamma}}\left\langle\mathbb{H}\left(g_{\varepsilon}^{H}\right), \mathbb{H}^{-1} A\right\rangle= & \varepsilon\left\langle\partial_{t} g_{\varepsilon}^{H}, A^{\prime}\right\rangle+\varepsilon^{1-\gamma}\left\langle v \cdot \nabla_{x} g_{\varepsilon}^{H}, A^{\prime}\right\rangle-\varepsilon^{\beta-\gamma-1}\left\langle\Gamma\left(g_{\epsilon}^{H}\right), A^{\prime}\right\rangle \\
& -\varepsilon^{m+q-\beta-\gamma}\left\langle\mathcal{H}_{1}^{\mathrm{HL}}\left(g_{\epsilon}^{\mathrm{HL}}\right), A^{\prime}\right\rangle-\varepsilon^{q-\gamma}\left\langle\mathcal{H}_{2}^{\mathrm{HL}}\left(g_{\epsilon}^{\mathrm{HL}}\right), A^{\prime}\right\rangle \\
& -\varepsilon^{m+q-\gamma}\left\langle\Gamma_{2}^{\mathrm{HL}}\left(g_{\epsilon}^{\mathrm{HL}}\right), A^{\prime}\right\rangle
\end{aligned}
$$

and

$$
\begin{aligned}
\frac{1}{\varepsilon^{\gamma}}\left\langle\mathbb{H}\left(g_{\varepsilon}^{H}\right), \mathbb{H}^{-1} B\right\rangle= & \varepsilon\left\langle\partial_{t} g_{\varepsilon}^{H}, B^{\prime}\right\rangle+\varepsilon^{1-\gamma}\left\langle v \cdot \nabla_{x} g_{\varepsilon}^{H}, B^{\prime}\right\rangle-\varepsilon^{\beta-\gamma-1}\left\langle\Gamma\left(g_{\epsilon}^{H}\right), B^{\prime}\right\rangle \\
& -\varepsilon^{m+q-\beta-\gamma}\left\langle\mathcal{H}_{1}^{\mathrm{HL}}\left(g_{\epsilon}^{\mathrm{HL}}\right), B^{\prime}\right\rangle-\varepsilon^{q-\gamma}\left\langle\mathcal{H}_{2}^{\mathrm{HL}}\left(g_{\epsilon}^{\mathrm{HL}}\right), B^{\prime}\right\rangle \\
& -\varepsilon^{m+q-\gamma}\left\langle\Gamma_{2}^{\mathrm{HL}}\left(g_{\epsilon}^{\mathrm{HL}}\right), B^{\prime}\right\rangle
\end{aligned}
$$

Hence, in the limit, in the case $0<\gamma=\beta<1$, it remains only the terms in $\Gamma$ in equations (4.29) and (4.30). Hence, we get

$$
\begin{array}{lll}
\left\langle\Gamma^{\mathrm{H}}\left(g_{\varepsilon}^{\mathrm{H}}\right), A^{\prime}\right\rangle & \underset{\varepsilon \rightarrow 0}{\longrightarrow} & \left\langle\Gamma^{\mathrm{H}}\left(g_{0}^{\mathrm{H}}\right), A^{\prime}\right\rangle=\left\langle\mathbb{H}\left(g_{0}^{\mathrm{H}}\right)^{2}, A^{\prime}\right\rangle=\left\langle\left(g_{0}^{\mathrm{H}}\right)^{2}, A^{\prime}\right\rangle=-B(u) \\
\left\langle\Gamma^{\mathrm{H}}\left(g_{\varepsilon}^{\mathrm{H}}\right), B^{\prime}\right\rangle & \underset{\varepsilon \rightarrow 0}{\longrightarrow} & \left\langle\Gamma^{\mathrm{H}}\left(g_{0}^{\mathrm{H}}\right), B^{\prime}\right\rangle=\left\langle\mathbb{H}\left(g_{0}^{\mathrm{H}}\right)^{2}, B^{\prime}\right\rangle=\left\langle\left(g_{0}^{\mathrm{H}}\right)^{2}, B^{\prime}\right\rangle=-5 \theta u
\end{array}
$$

where we have use the fact that, for any $g_{0}^{\mathrm{H}} \in \mathcal{N}$, it holds that

$$
\Gamma\left(g_{0}^{\mathrm{H}}\right)=-\mathbb{H}\left(\left(g_{0}^{\mathrm{H}}\right)^{2}\right) .
$$

Now, since $\operatorname{div} u=0$, we find that

$$
\nabla \cdot(\theta u)=u \cdot \nabla \theta, \quad \nabla \cdot B(u)=u \cdot \nabla u-\frac{1}{3} \nabla|u|^{2} .
$$

In view of $(4.31,(4.32)$ and $(4.34)$, the function $u$ and $\theta$ are solution to the following equations

$$
\left\{\begin{array}{l}
\frac{\partial u}{\partial t}+u \cdot \nabla u+\nabla p^{\mathrm{H}}=0 \\
\frac{\partial \theta}{\partial t}+u \cdot \nabla \theta=0 .
\end{array}\right.
$$

This completes the proof of the Theorem 3.1.

\section{Research Perspectives}

The present paper has been devoted to generalize the asymptotic analysis proposed in [4]. According we summarized our results in the table 1.

The main research perspectives is concerned with the derivation of the "ghost effects" shown recently by Sone [25], [23], [26]. According to the ghost effects, the system shows that the flow of the mixture vanishes in the continuum limit, but the vanishing flow gives a finite effect on the behavior of the mixture in this limit.

It is worth stressing that from the mathematical viewpoint, the ghost effect means that the equations governing the time evolution of the density and the temperature depends on the scaled momentum velocity even though the macroscopic velocity tends to zero. An 


\begin{tabular}{|c|c|c|c|c|}
\hline$\gamma$ & $\beta$ & $\boldsymbol{m}$ & & \multicolumn{1}{c|}{ Hydrodynamic limit } \\
\hline$\gamma=0$ & $\beta=0$ & $m=1$ & & $\begin{array}{l}\text { Solution near absolute Maxwellian } \\
\text { Compressible Euler for H and } \\
\text { Incompressible Navier-Stokes for L } \\
\text { Stokes equation for L }\end{array}$ \\
\hline$\gamma>0$ & $\beta=0$ & $m=1$ & & $\begin{array}{l}\text { Compressible Euler for H } \\
\text { Incompressible Navier-Stokes for L }\end{array}$ \\
\hline$\gamma=0$ & $\beta>0$ & $m>1$ & & $\begin{array}{c}\text { Acoustic system for H } \\
\text { Incompressible Navier-Stokes for L }\end{array}$ \\
\hline $0<\gamma$ & $=\beta<1$ & $m=1$ & & $\begin{array}{c}\text { Incompressible Euler for H } \\
\text { Incompressible Navier-Stokes for L }\end{array}$ \\
\hline
\end{tabular}

TABLE 1. The various fluid dynamic regimes of the Boltzmann mixture system in terms of the dimensionless parameters

interesting phenomenon of the ghost effect is that the flow moves from the low temperature to the high one when the gas is very rarified, while in the Euler or Navier-Stokes level, the flow moves from the high temperature to the low one, which is well known in the heat flow.

Moreover we are interested in showing that the sequence: Euler equations, Navier-Stokes equations, Burnett equations, etc. breaks down at the Burnett level since these equations are ill-posed [5]. In fact, it is well known (see [5], [6], [7]) that the Burnett hydrodynamic equations violate the basic physics behind the Boltzmann equation.

The rigorous proofs are in progress and the results will be presented in due course.

\section{REFERENCES}

[1] P. Andries, K. Aoki, and B. Perthame. A consistent BGK-type model for gas mixtures. J. Statist. Phys., 106(5-6):993-1018, 2002.

[2] K. Aoki, C. Bardos, and S. Takata. Knudsen layer for gas mixtures. J. Statist. Phys., 112(3-4):629-655, 2003.

[3] C. Bardos, F. Golse, and C. Levermore. Fluid dynamic limits of kinetic equations. I. Formal derivations. J. Statist. Phys., 63(1-2):323-344, 1991.

[4] C. Bianca and C. Dogbe. On the Boltzmann gas mixture equation: Linking the kinetic and fluid regimes. Communications in Nonlinear Science and Numerical Simulation, 29:240-256, 2015.

[5] A. V. Bobylev. On the Chapman-Enskog and Grad methods for solving the Boltzmann equation. Dokl. Akad. Nauk SSSR, 262(1):71-75, 1982.

[6] A. V. Bobylev. Generalized Burnett hydrodynamics. J. Stat. Phys., 132(3):569-580, 2008.

[7] A.V. Bobylev and A. Windfäll. Boltzmann equation and hydrodynamics at the Burnett level. Kinet. Relat. Models, 5(2):237-260, 2012.

[8] R. E. Caflisch. The fluid dynamic limit of the nonlinear Boltzmann equation. Comm. Pure Appl. Math., 33(5):651-666, 1980.

[9] C. Cercignani. The Boltzmann equation and its applications, volume 67 of Applied Mathematical Sciences. Springer-Verlag, New York, 1988.

[10] S. Chapman and T. G. Cowling. The Mathematical Theory of Non-uniform Gases. Cambridge University Press, Cambridge, 1939.

[11] C. Dogbe. Diffusion approximation for a Knudsen gas in a thin domain with reflexive chaotic law. Bull. Austral. Math. Soc., 63(3):497-514, 2001.

[12] C. Dogbe. Fluid dynamic limits for gas mixture. I. Formal derivations. Math. Models Methods Appl. Sci., 18(9):1633-1672, 2008.

[13] J. Ferziger and H. G. Kaper. Mathematical theory of transport processes in gases. North-Holland Pub. Co., Amsterdam, 1972. Distributed in the U.S. by American Elsevier Pub. Co., New York. 
[14] R. T. Glassey. The Cauchy problem in kinetic theory. Society for Industrial and Applied Mathematics (SIAM), Philadelphia, PA, 1996.

[15] E. Goldman and L. Sirovich. Equations for Gas Mixtures. Physics of Fluids (1958-1988), 10(9):19281940, 1967.

[16] H. Grad. On the kinetic theory of rarefied gases. Comm. Pure Appl. Math., 2:331-407, 1949.

[17] J. O. Hirschfelder, C. F. Curtis, and R. B. Bird. Molecular Theory of Gases and Liquids. Wiley, New York, 1954.

[18] M. N. Kogan. The equations of the kinetic theory of gases. In Rarefied Gas Dynamics, pages 29-104. Springer US, 1969.

[19] A. De Masi, R. Esposito, and J. L. Lebowitz. Incompressible Navier-Stokes and Euler limits of the Boltzmann equation. Comm. Pure Appl. Math., 42(8):1189-1214, 1989.

[20] N. Masmoudi. Hydrodynamic limits of the Boltzmann equation. In Transport in transition regimes (Minneapolis, MN, 2000), volume 135 of IMA Vol. Math. Appl., pages 217-230. Springer, New York, 2004.

[21] A. Mellet. Diffusion limit of a non-linear kinetic model without the detailed balance principle. Monatsh. Math., 134(4):305-329, 2002.

[22] Y. Sone. Asymptotic theory of a steady flow of a rarefied gas past bodies for small knudsen numbers. In René Gatignol and Soubbaramayer, editors, Advances in Kinetic Theory and Continuum Mechanics, pages 19-31. Springer Berlin Heidelberg, 1991.

[23] Y. Sone. Continuum gas dynamics in the light of kinetic theory and new features of rarefied gas flows. Adv. Mech., 27(3):389-404, 1997.

[24] Y. Sone. Molecular gas dynamics. Modeling and Simulation in Science, Engineering and Technology. Birkhäuser Boston, Inc., Boston, MA, 2007. Theory, techniques, and applications.

[25] Y. Sone, K. Aoki, S. Takata, H. Sugimoto, and A. V. Bobylev. Inappropriateness of the heat-conduction equation for description of a temperature field of a stationary gas in the continuum limit: examination by asymptotic analysis and numerical computation of the Boltzmann equation. Phys. Fluids, 8(2):628638, 1996.

[26] S. Takata and K. Aoki. The ghost effect in the continuum limit for a vapor-gas mixture around condensed phases: asymptotic analysis of the Boltzmann equation. Transport Theory Statist. Phys., 30(2-3):205237, 2001. The Sixteenth International Conference on Transport Theory, Part I (Atlanta, GA, 1999).

(Carlo Bianca) Laboratoire de Physique Statistique, Sorbonne Universités, Ecole Normale Supérieure, 75231 Paris cedex 05, France, and CNRS, UMR 8550 LPS, Paris, France. E-mail address: bianca.carlo@gmail.com

(Christian Dogbe) Department of Mathematics, Université de Caen, Lmno, CNRS, UmR 6139, 14032 Caen Cedex, France. E-mail address: christian.dogbe@unicaen.fr 